\section{Heavy Ion Accelerators for Inertial Fusion}

C. Rubbia, the Director-General of CERN, has contributed extensively to the design of accelerators for inertial fusion. We summarise here several recent presentations.

Particle accelerators appear as the most promising drivers for a fusion power reactor based on inertial confinement. In particular, accelerators are unique in having a high driver efficiency, a high repetition rate and high durability [1]. In addition, accelerator technology is well-developed and extremely reliable - crucial points for an industrial power reactor based on the indirect drive approach where a capsule containing a deuterium-tritium fuel mixture is inserted inside a blackbody hohlraum casing. Filamentary radiators (some 6 to 8 in number [2]) instead of one difficultto-engineer radiator protrude through the casing wall (Fig. 1). These convert the kinetic energy of impacting ions into soft $X$-rays which ablate the inner wall of the casing during multiple reflections, thus giving rise to a symmetric implosion of the capsule (see cover illustration).

The very stringent requirements for indirectly driven ICF pellets make the design of the accelerator complex a difficult task. The complex must deliver 5-10 MJ of energy within $10-15 \mathrm{~ns}$ and the beam spot for indirect drive must be small enough ( $\approx 1 \mathrm{~mm}$ in diameter) to produce energy depositions $E$ in the radiators of the target pellets of $10^{16}-10^{17} \mathrm{~W} / \mathrm{g}$ corresponding to a peak power of 500-1000 TW. To minimise space charge effects one must manipulate ions with as few charges as possible. The charge state $q$ is set to 2 , this being the lowest value for which the most promising technique to stack ions in a storage ring, namely photo-ionisation (see below), can be applied.

A practical scheme for the accelerator complex to produce heavy ion beams with

the required properties consists of a linear single-pass accelerator followed by a set of storage rings. A type of accelerator which would satisfy the efficiency requirements and of well-known performance is the conventional linac, where currents in the range 50-100 mA for singly ionised heavy ions are possible. A $10 \mathrm{GeV}$ accelerator and an energy transported by the tion $\Delta t$ of $10 \mathrm{~ms}$. Since $\Delta \tau$ the duration of the pulse on the pellet must be $\approx 10 \mathrm{~ns}$, a compression factor of $10^{6}$ is needed. Such compression factors $\Delta t / \Delta \tau$ can be achieved by having a large number of beams focussed simultaneously on the pellet. This feature is also required for uniformity of driving.

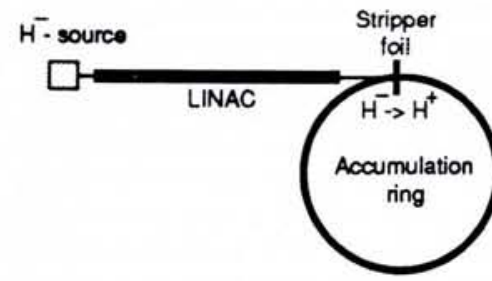
beam of $10 \mathrm{MJ}$ would require a pulse dura-

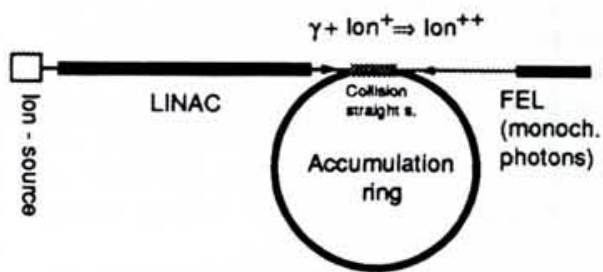

Fig. $2-\mathrm{H}$ - injection with a stripping foil (a) compared with (b) heavy ion injection using photo-ionisation by UV light from a free-electron laser.

\section{Circumventing the Liouville Theorem Photo-ionisation}

Methods such as stochastic and electron cooling, to minimise the dilution of phase space, do not extrapolate to the ICF requirements as they are too slow when one is dealing with $10^{15}$ to $10^{16}$ singly ionised heavy ions. Closest to to the envisaged application is so-called negative ion injection invented by Budker and nowa-

\section{Phase Space Conservation and the Non-Liouvillian Method}

Focussing on a small pellet across an ICF reactor chamber requires beams with a small angular divergence. In the language of accelerator physics, such beams must have a small emittance, i.e., phase space volume defined in terms of position and momentum coordinates. According to canonical mechanics, the exact phase space volume of an ensemble of particles is an invariant (Liouville's theorem).

In accelerators, it is more appropriate to speak of the "effective emittance" which takes into account filamentation of phase space, and thus an increase in the phase space volume effectively used. Such dilution occurs at injection into a ring, during muliturn stacking, or during RF bunching and bunch compression. Keeping phase space dilution to a minimum is a major challenge in heavy ion fusion (HIF) research.

Methods of circumventing Liouville's theorem have therefore been discussed since the earliest days of HIF. $\mathrm{H}^{-}$stripping injection (by a foil) into high-current proton storage rings has been common since the late-1960's. For heavy ions, the idea is to use photons from an intense laser beam to strip singly to doubly charged ions at injection. Due to the different deflection in a magnetic field, the phase space region of the injected beam can be merged with that of the circulating beam. The process is repeated many times and each newly injected turn enters the phase space volume of the previous one. Liouville's theorem is invalid due to the change of particle species. days almost universally used in high-energy proton machines. A thin stripping foil is used to convert $\mathrm{H}^{-}$ions into protons (Fig. 2a) and the corresponding phase compression is only limited by scattering in the foil, thus eluding the conditions set by the Liouville theorem. Since de facto the ionisation is produced by the "photons" Fourier transformed from the Coloumb field of the stripper atoms as seen by the moving beam, one could think to extend the concept to heavy ions with the help of a "smart foil" made of an intense and monochromatic beam of UV light.

The most promising and simplest way of accumulating the required current in storage rings is therefore photo-ionisation stacking (Fig. 2b). Intense UV light ionises the beam from the linac from singly to doubly charged ions. After storage there is no further effect of the UV light on the beam as long as the energy of the photons is less than two to three times the ionisation potential. Single to double ionisation cross-sections for the heaviest ions are typically $\approx 10^{-16} \mathrm{~cm}$ corresponding to a light wavelength of $50-100 \mathrm{~nm}$. However, the exceedingly large $\left(>2.10^{-15} \mathrm{~cm}^{2}\right)$ double ionisation cross-sections of a resonant nature reported for $\mathrm{Ba}$ simplify the design of the light source.

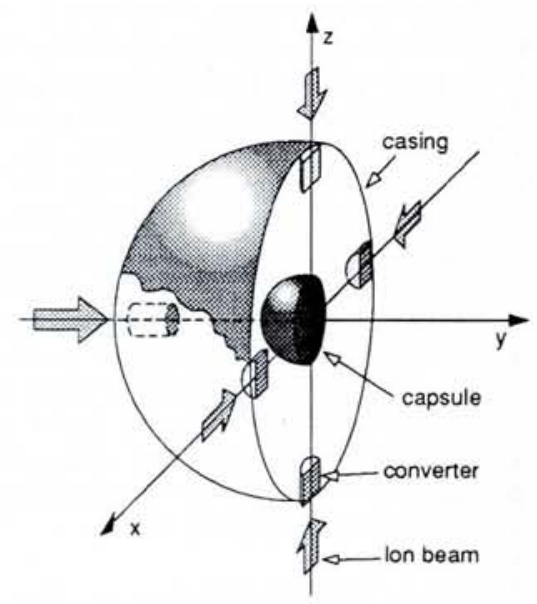

Fig. 1 - A pellet for indirectly driven heavy ion inertial fusion. A capsule containing the $D-T$ fuel mix is contained inside a casing that acts as a blackbody hohlraum. lons from many beam-straws strike the convertors (shown as filamentary radiators) embedded in the casing and the ion energy is converted into soft $X$-rays. 


\section{Beam-straws}

A second concept which further simplifies the design of an accelerator complex for fusion is the use of a very large number (> 1000) of beamlets - or beam-straws - all focussed on the same spot on the pellet. This subdivision elegantly circumvents the problems of space charge and exploits the non-Liouvillian nature of the stopping power of the pellet. The ultimate current on the target can be maintained by having rather low currents in the beamstraws for which space charge effects are manageable with small emittances. Thus, following appropriate stacking and RF compression, several bunches from each of the extracted beams are spliced into separate beam-straws and transported to the inertial fusion combustion chamber.

Appropriate devices are needed in order to handle the unprecedented number of beam-straws. The key element is the idea of a magnetic focussing lattice, namely a repetitive (elementary) electrode configuration generating a magnetic quadrupole field with a major fraction of the volume free for the passage of beams (Fig. 3).

\section{Window of Feasibility}

Limitations due to space charge effects are critical in defining the parameters of the proposed scheme and can be used to determine a fairly narrow feasibility window within the delineated general scenario. Among the many different known types of instabilities which may plague a strong focussing beam storage and transport system, it turns out that the most relevant to the present application are those associated with transverse motion. Longitudinal effects can be, in general, controlled during storage, which must be kept short as the total manipulation time must be less than a few tens of milli-

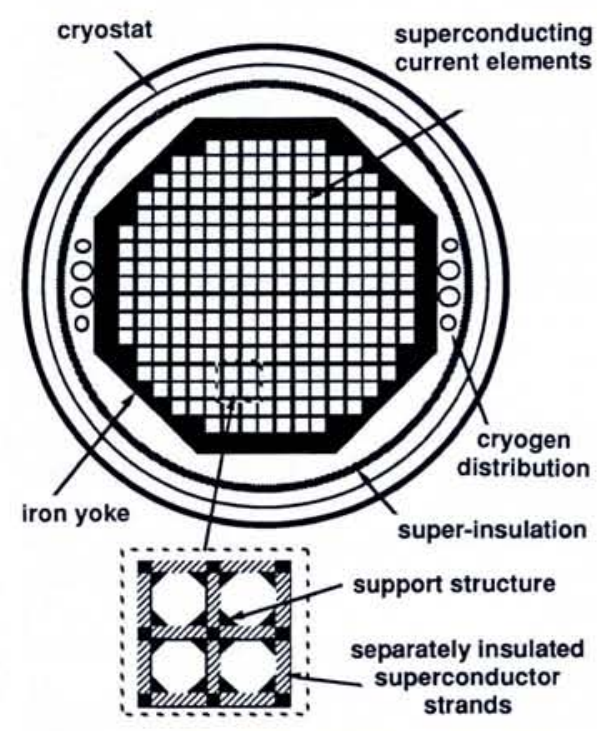

Fig. 3 - A section through a superconducting quadrupole lattice (256 elements) for focussing multiple beam-straws.

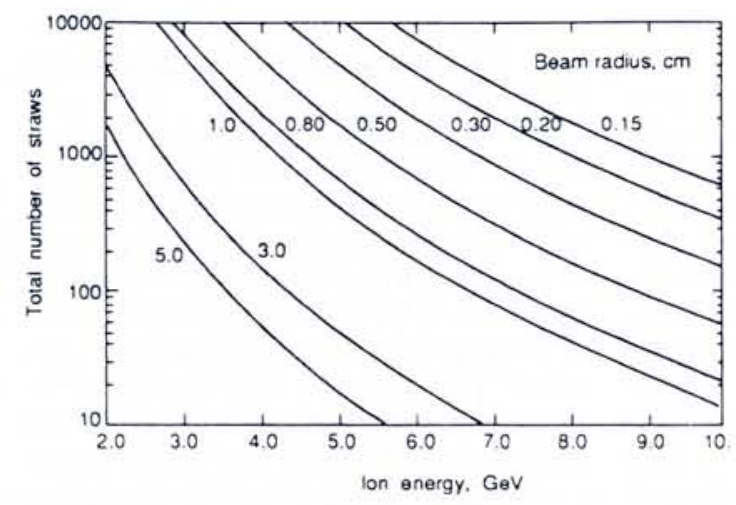

Fig. 4-The calculated number of beam-straws needed for a total power of $500 \mathrm{TW}$ with a 0.1 sterad total solid angle. The number is plotted as a function of the ion (Ba) kinetic energy for various beam focal radii (in $\mathrm{cm}$ ).

seconds to avoid elastic intra-beam scattering from changing the charge state $q$.

The three critical areas relate to:

- the high current stored in the beams of the storage rings during and particularly at the end of the stacking process;

- the last phase of bunch compression, just before beams are directed towards the pellet in the single pass through a long transport section;

- the convergence towards the pellet inside the free space of the reactor.

Definition of the parameters of the complex can best be done backwards, namely setting first the conditions resulting from beam transport and adapting the storage rings and the linac characteristics to the space charge requirements in the final beam transport.

\section{Final transport}

The high currents needed to implode the pellet produce a space charge defocusing which is comparable to the focussing forces of the beam transport channel. All sorts of instabilities may develop under such circumstances. For a discontinuous focussing system, there is an emittance growth produced by resonance effects due to the periodicity of the focus. These effects occur for critical phase advances $\mu$ per focussing period of the betatron function describing the transverse oscillations of the beam particles.

Conservative estimates [3] indicate that to avoid fourth and higher order instabilities, a beam transport section consisting of evenly space quadrupoles requires $\mu$ in the presence of space charge to be larger than $0.4 \mu_{0}$, where $\mu_{0}$ is the advance in the absence of space charge effects. Avoiding pronounced third-order instabilities requires $\mu_{0}<60^{\circ}$.

Consider a beam channel made up of simple FODO (focus - straight-defocusstraight) cells comprising quadrupole magnets separated by dipole magnets. The maximum number of ions $N_{\text {straw }}$ per unit of length / which can then be transported in a FODO channel with a given $\mu$ is proportional to the space charge limited minimum emittance $\varepsilon_{\text {straw }}$ of the beamstraw. The emittance is related to the space charge limited spot radius $\langle r\rangle$ and the beam angular divergence $\langle\theta\rangle$ in the reactor chamber. The latter is in turn related to the solid angle $\left.\Omega_{\text {straw }}=\pi<\theta\right\rangle^{2}$ occupied by the beam-straws. The total solid angle subtended by a large number $S$ of beam-straws converging on a given radiator in the pellet is given by $\Omega_{\text {tot }}=$ $S \Omega_{\text {straw. }}$. As these openings let energy from the explosion of the pellet escape, they must be kept to a minimum so $\Omega_{\text {tot }}$ is considered as an input parameter.

It has been shown [4] that all the features of a beam limited by space charge in the final transport can be derived uniquely in terms of a parameter $\Gamma$ having the form $\Gamma=a T^{-n}(E / \Delta \tau)$

where $a$ and $n$ are constants depending on the type on ion and $T$ is the kinetic energy of the ions. For instance, it turns out that the space charge limited spot radius $\langle r\rangle$ $\sim S^{-1 / 2}$, underlining the non-Liouvillian nature of the energy losses in the absorber and the advantage of keeping the number of straws large.

The beam optics inside the reactor chamber are determined totally by $\beta^{*}=$ $\langle r\rangle \mid\langle\theta\rangle$, the beta function at the focal point, since $\beta_{(z)}=\beta^{*}+z^{2} / \beta^{*}$ with the focal point at $z=0$ in the longitudinal direction $z$. Consider $N$ identical focal points, each with $S$ beam-straws focussed on a spot in one of several identical radiators. Eq. (1) also holds for the global $\Gamma_{\text {all }}$ and for the total energy on the pellet. For a correspondingly increased solid angle $\Omega_{\text {all }}=N \Omega_{\text {tot }}$ we have

$$
N S=\Gamma_{\text {all }}^{2} / \pi \Omega_{\text {all }}<r>^{2} .
$$

The total number of beam-straws on the pellet NS can thus be calculated for different ion energies $T$ and beam focal radii. Two possibilities (Fig. 4) emerge for $E / \Delta \tau$ $=500 \mathrm{TW}$, a solid angle $\Omega_{\text {all }}=0.1 \mathrm{sterad}$ and a reasonable number of straws on the order of a few times $10^{3}$. In one alternative (best suited to filamentary radiators), the beam spots are focussed down to about $1-2 \mathrm{~mm}$ radius. This corresponds to the high energy option for beams of either $\mathrm{Ba}$ or $\mathrm{Bi}$ of $8-10 \mathrm{GeV}$, where the range of ions in the absorber is considerable and a thick absorber is necessary. The second alternative - more suited to direct heating of a hohlraum - corresponds to a focal point of $\geq 5 \mathrm{~mm}$ radius and is best implemented with low energy beams with the shortest possible range (i.e., 4-5 GeV). 


\section{Storage rings}

Now that the parameters of the final focus have been narrowed down we can consider the requirements of the storage rings. Neglected at this stage are phase space blow-up and particle losses in these manipulations, which simply consist of a longitudinal bunch compression followed by extraction and transfers. Bunch coalescence is not used so both the number of bunches and the total number of particles/bunch are conserved. The total number of rings $n_{\text {rings }}$ is related to the number of bunches $h$ which can be stored in each ring, since $S=h n_{\text {rings }}$. The stability limit in the transverse plane is determined by the maximum incoherent tuning shift $\Delta Q$. This sets a limit to $N_{\text {straw }}$ the number of ions stored in each straw (bunch).

The total straw length at the final focus that each ring can supply is given by [3]

$$
I_{\text {straw }} h=16 \pi B \Delta Q / K^{1 / 2} Q^{\prime}
$$

where $I_{\text {straw }}$ is the effective length of the beam straw at the pellet, $B$ is the bunching factor, $K$ is the external focussing constant, and $Q^{\prime}$ is a function tabulated in [2]. A typical achievable value for the total straw length is $25.12 \mathrm{~m}$. For an ion speed $\alpha c, l_{\text {straw }}=\alpha c \Delta \tau=0.9 \mathrm{~m}$, suggesting that $h=16$ if it is to remain a multiple of 2. Hence, some $S / 16$ rings are needed and the number of beam splittings which are optimal is rather rigidly determined by space charge considerations.

\section{Photo-ionisation driven stacking}

Stacking for photo-ionisation can be induced along a straight section of the storage ring for the doubly ionised particles which we assume for simplicity to be a matched FODO quadrupole magnetic focussing lattice. Injection occurs when the singly ionised beam from the linac collides head-on with an intense and monochromatic UV light beam (most appropriately produced by a free-electron laser or FEL). Bending magnets at the beginning and the end are used to separate the orbit of the singly ionised beam of the linac from the equilibrium orbit of the stored beam. There is a difference in parameters of the FODO channel between the singly and doubly charged beams and both beams must be separately matched outside the common straight section.

If a parallel optical beam of uniform intensity is matched to the ion beam, it is possible to calculate the power needed for a given ionisation efficiency. For $90 \%$ efficiency with $\mathrm{Ba}$ ions, the power is 29.28 $\mathrm{kW}$ if collision takes place over two FODO periods (the light intensity would be much larger for non-resonant ions such as $\mathrm{Bi}$ ). An emittance blow up of $20-30 \%$ must be allowed for owing to the difference in the betatron function between singly and doubly charged $(\mathrm{Ba})$ ions. Second, in the case of $\mathrm{Ba}$, as the resonance peaks are very narrow both the ion speed and the photon energy must be defined with sufficient precision so as not to exceed the resonance width.

The requirements of the light source indicate that the FEL must be driven by an electron linac where the required wavelength $(80 \mathrm{~nm})$ can be produced by a twostage device to permit the use of an electron beam with less stringent properties. One needs a peak bunch (electron) current of $\approx 200 \mathrm{~mA}$ at $250 \mathrm{MeV}$ which is within the range of special state-of-the art photocathodes and the FEL would deliver a $1 \mathrm{MW}$ peak power after $14 \mathrm{~m}$ of undulator and saturate at $69 \mathrm{MW}$ after $21 \mathrm{~m}$. As the light power for photo-ionisation is considerably smaller, the average linac power current need only be $\approx 20 \mathrm{~mA}$ for Ba ions. tude in the choice of focal strength at focus, chromatic corrections are modest, and full convergence on the pellet can be ensured even from large angles as the ion range in the absorber is very short. Beam dumps and $S$-shaped magnetic deflection of the beam-straws would ensure a negligible amount of radiation escapes from the reactor via the paths of the beamstraws.

\section{Conclusions}

A number of novel techniques developed in high energy physics may be beneficial to heavy ion induced confinement applications. In particular, non-Liouvillian stacking of the ion beams for a fusion driver appears feasible for $\mathrm{Bi}$ and very

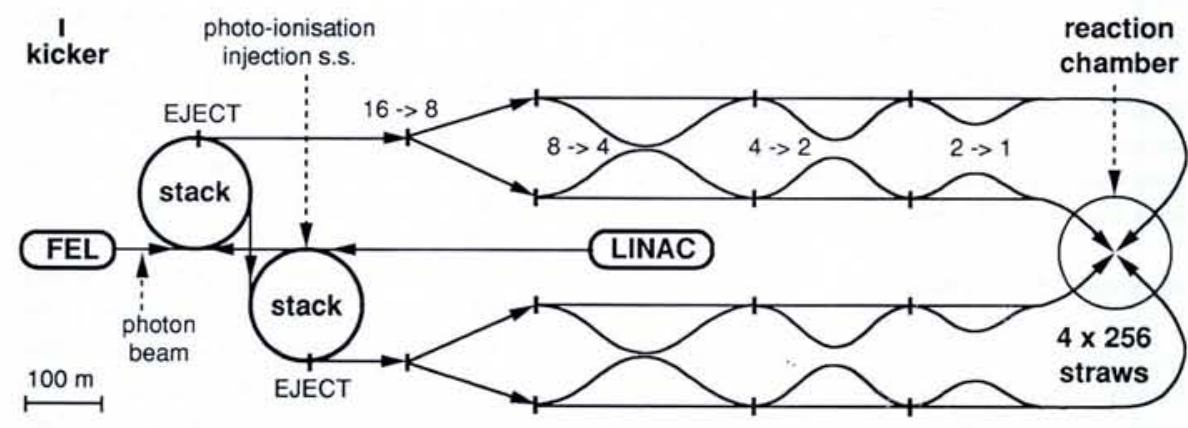

Fig. 5 - General layout of an accelerator complex (1.5 $\mathrm{km}$ in length) for four filamentary radiators. A free-electron laser is used to photo-ionise linac accelerated heavy ions that are loaded in two counter-rotating stacked ring structures ready for simultaneous extraction and transport to the reaction chamber.

\section{A Practical Scheme}

The general layout of the accelerator complex for producing $\mathrm{Ba}$ beams at 10 $\mathrm{GeV}$ intended for indirectly driven ICF is shown in Fig. 5 . The beam of singly ionised ions from a 50-100 mA linac is accumulated (with photo-ionisation) in a relatively large number of storage rings with a stacked configuration sharing the main elements of the magnetic structure (as at the CERN-PS booster). Each ring would be filled in series from the linac. RF cavities on straight sections maintain beam bunching during stacking with a relatively low bunching factor to accumulate the largest possible number of ions. Shortly before extraction, the bunches are compressed by adjusting the RF parameters and ions are extracted simultaneously from all rings, as a "one-turn" extraction in one or several channels, using a full-aperture set of kickers with a rise time faster than the bunch spacing. Bunches of each beam are then divided into two equal half-strings and the procedure repeated to give the required number of beam-straws on the pellet.

The final focus onto the pellet is the densest part of the beam-straw geometry and resembles the low- $\beta$ crossing in a high-energy collider. There is a large lati- favourable for $\mathrm{Ba}$, but it requires the development of a free-electron laser that has only been designed on paper.

A bold extension of the multiple-ring technique of CERN's PS booster to ion stacking and $\approx 1000$ beam-straws would permit the design of rings with individual beams in a well-tested domain of operation. Existing and proven techniques should allow the conditions for indirectly driven ignition to be reached. $\mathrm{Ba}$ ions of 8-10 GeV corresponding to the "high likelihood" case for high-gain pellet implosion with 4 or 8 filamentary radiators can be obtained from a compact ring configuration fed by a standard $50 \mathrm{~mA}$ linac.

\section{REFERENCES}

[1] Rubbia C., presented at the Int. Conf. on Evolution in Beam Applications, Takasaki, Japan, 1991.

[2] Murakami M., Meyer-ter-Vhen J. and Ramis R., J. X-Ray Sci. \& Tech. 2 (1990) 127.

[3] Hofmann I. et al., Particle Accelerators 13 (1983) 145.

[4] Rubbia C., Proc. of "Drivers for Inertial Confinement Fusion", Osaka (1991) 47.

[5] Bonifacio R., et al., unpublished research, INFN, Milan (1990). 